\title{
LITTER-MATE ASSAYS OF PERTUSSIS VACCINE
}

\author{
BY J. O. IRWIN AND A. F. B. STANDFAST \\ The Medical Research Councils' Statistical Research Unit, London School \\ of Hygiene and Tropical Medicine, and the Lister Institute \\ of Preventive Medicine, Elstree, Herts
}

It is well known that in biological assays, in which the animal response is measureable, increased accuracy is often obtained by using litter-mates. One member of each litter is placed on each dose of the standard and unknown preparations. The increased accuracy arises from the fact that litter-mates are frequently less variable in response than animals which are not isogenic. The same principle should apply to assays using a quantal response, in which the probit technique is usually used, but it is not immediately obvious how the necessary allowance for litter-mates should be made in the statistical analysis. One of us (Irwin, 1950) described how this could be done, but at the time there were no suitable data to analyse. The data are now available of eight assays of two pertussis vaccines (K and $H)$ using littermates, carried out by Standfast in 1950 by the mouse intra-cerebral method.

\section{MATERIAL AND METHODS}

Mice. The mice were from an inbred strain which had been maintained for many generations by brother-sister mating. For the experiments reported here only those litters which contained six or more mice (at weaning) were used. On weaning, the mice from each litter were separated into two cages, one of males and one of females, and kept until they weighed 14-18 g. Each cage was, of course, carefully labelled with the details of the litter, sex, etc.

When the mice weighed 14-18 g. 15 litters of 6 or more were divided into single cages to make 6 sets of 15 mice, one from each litter, and these were used for the test proper (see Table 1). The extra mice from the larger litters were divided as evenly as possible into 4 sets and the numbers made up to 15 with mice from litters of identical breeding; these extra mice were used for the titration of the challenge dose. Though there was a fair number of litters of 10 mice it was never possible to collect 15 litters of 9 or 10 mice. Thus, only two vaccines can be compared at one time by this method.

The amount of work necessary in recording and maintaining these mice means that this type of test is hardly worth while unless the results are very superior.

Protection tests. Two vaccines $\mathrm{K}$ and $\mathrm{H}$ were compared in each assay; they were given in the doses $80 \times 10^{6}, 400 \times 10^{6}$ and $2000 \times 10^{6}$ of vaccine and challenged intracerebrally 10 days later with 50,000 Haemophilus pertussis strain 18-323, by the method of Kendrick, Eldering, Dixon \& Misner (1947). 


\section{STATISTICAL ANALYSIS}

The essential data are given in Table 1. The method of analysis is as follows:

(1) Standard probit analysis is used to find the best fitting straight line connecting the probit, corresponding to the proportion of mice surviving, with the logarithm of the dose. This straight line has the equation $Y=\bar{y}+b(x-\bar{x})$, where $x=\log$ dose, and $Y, \bar{y}$ are the probits corresponding to the expected and observed responses at $\log$-dose $x$.

The working probit corresponding to $\log$-dose $x$ is $Y+p / Z-P / Z$, with a weighting factor $Z^{2} / P Q$. Thus the working probit for zero response is $Y-P / Z$, for $100 \%$ response it is $Y+Q / Z$, where $Q=1-P$; and for each animal we have a working probit of $Y-P / Z$ or $Y+Q / Z$ according as it dies or survives, with weight $Z^{2} / P Q$.

(2) A weighted analysis of variance of the working probits for all animals is carried out, so that the mean squares due to 'doses', 'litters' and 'doses $\times$ litters' can be isolated. If the second mean-square is significantly greater than the third, there are significant differences between litters and there is a gain in accuracy from using litter-mates. The third mean square then provides an estimate of experimental error. The expectation of the second mean square is always unity (provided that the probit-log-dose relation is in fact linear); the expectation of the third mean square is unity if there is no correlation between litter-mates and less than unity if there is a positive correlation between litter-mates. This method was applied in three ways.

(i) Separate straight lines were fitted for each vaccine, $\mathrm{K}$ and $\mathrm{H}$, in each assay. The corresponding working probits were then computed for each animal and the error mean square obtained by analysis of variance. Estimates of the two E.D. 50's and their errors were then obtained.

(ii) Parallel straight lines were fitted for the vaccines $\mathrm{K}$ and $\mathrm{H}$ in each assay, the rest of the procedure being the same as before. Estimates of the two E.D. 50's were again obtained as well as the potency ratio and an estimate of its error.

(iii) The errors of the estimates of slope obtained by methods (i) and (ii) were very large. This resulted in very large errors for the estimate of potency. It was found, however, that there were no significant differences in the estimates of slope obtained from all the tests, nor were there any significant departures from linearity. Thus it was justifiable to compute and use an overall estimate of slope. When this had been done, the estimates of the two E.D. 50's, the potency ratio and their errors were obtained as before.

The test performed on 12 June 1950 (see Table 1) was not analysed statistically. It is clear that the percentages of survivors on the two lower doses were anomalously high. In the test on 22 May 1950, no estimate of slope could be obtained for $\mathrm{H}$ owing to the zero responses to the two lower doses, and the slope for $\mathrm{K}$ was not significant. Method (i) above was not therefore applied in this test, nor, for similar reasons, to the tests carried out on 11 July 1950 or on 18 July 1950. Methods (ii) and (iii) were not applied to the last test, where all the animals receiving $\mathrm{H}$ died. 
Table 1

\begin{tabular}{|c|c|c|c|c|c|c|c|c|c|c|c|c|c|c|c|c|c|c|c|}
\hline \multirow[b]{2}{*}{ Date of test } & \multirow[b]{2}{*}{ Vaccine } & \multirow{2}{*}{$\begin{array}{l}\text { Dose } \\
\times 10^{6} \\
\text { orgs. }\end{array}$} & \multirow{2}{*}{$\begin{array}{l}\text { Mouse } \\
\text { no. }\end{array}$} & \multicolumn{15}{|c|}{ Litter } & \multirow{2}{*}{$\begin{array}{c}\text { No. } \\
\text { surviving }\end{array}$} \\
\hline & & & & 1 & 2 & 3 & 4 & 5 & 6 & 7 & 8 & 9 & 10 & 11 & 12 & 13 & 14 & 15 & \\
\hline \multirow[t]{5}{*}{ 11. iv. 50} & $\mathbf{K}$ & 80 & 1 & $\mathrm{D}$ & D & $\mathrm{D}$ & s & D & D & D & $\mathrm{D}$ & D & $\mathbf{s}$ & $\mathbf{s}$ & D & $\mathrm{D}$ & $\mathbf{s}$ & $\mathrm{D}$ & $4 / 15$ \\
\hline & & $\begin{array}{r}400 \\
2000\end{array}$ & $\begin{array}{l}2 \\
3\end{array}$ & $\begin{array}{l}\mathrm{S} \\
\mathrm{S}\end{array}$ & $\begin{array}{l}\mathrm{s} \\
\mathrm{s}\end{array}$ & $\begin{array}{l}\mathrm{s} \\
\mathrm{S}\end{array}$ & $\begin{array}{l}\mathrm{D} \\
\mathrm{S}\end{array}$ & $\begin{array}{l}\mathrm{D} \\
\mathrm{S}\end{array}$ & S & $\begin{array}{l}\mathrm{D} \\
\mathrm{S}\end{array}$ & $\begin{array}{l}\mathrm{D} \\
\mathrm{D}\end{array}$ & $\begin{array}{l}\text { S } \\
\mathrm{D}\end{array}$ & $\begin{array}{l}\mathrm{S} \\
\mathrm{S}\end{array}$ & $\begin{array}{l}\mathrm{D} \\
\mathrm{D}\end{array}$ & $\begin{array}{l}\mathrm{D} \\
\mathrm{D}\end{array}$ & $\begin{array}{l}\mathrm{D} \\
\mathrm{S}\end{array}$ & $\begin{array}{l}\mathrm{D} \\
\mathrm{s}\end{array}$ & $\begin{array}{l}\mathrm{D} \\
\mathrm{D}\end{array}$ & $\begin{array}{r}6 / 15 \\
10 / 15\end{array}$ \\
\hline & $\mathrm{H}$ & 80 & 4 & $\mathrm{D}$ & D & D & $\mathrm{D}$ & D & $\mathrm{D}$ & $\mathrm{D}$ & $\mathrm{D}$ & D & $\mathrm{D}$ & - & $\mathrm{D}$ & $\mathrm{D}$ & D & D & $0 / 14$ \\
\hline & & 400 & 5 & D & D & D & D & D & $\mathrm{s}$ & D & D & D & D & $\mathrm{s}$ & $\mathrm{D}$ & D & D & D & $2 / 15$ \\
\hline & & 2000 & 6 & - & $\mathrm{s}$ & $\mathbf{S}$ & D & D & D & s & $s$ & - & $\mathbf{s}$ & $\mathrm{s}$ & $\mathrm{S}$ & D & D & D & $7 / 13$ \\
\hline \multirow[t]{6}{*}{ 1. v. 50} & $\mathrm{~K}$ & 80 & 1 & D & D & D & D & D & - & D & $\mathrm{D}$ & D & D & D & D & D & $\mathrm{s}$ & D & $1 / 14$ \\
\hline & & 400 & 2 & $\mathrm{~s}$ & D & $\mathrm{s}$ & D & D & $\mathrm{s}$ & D & D & D & $\mathrm{D}$ & D & $\mathrm{D}$ & - & $\mathrm{s}$ & D & $4 / 14$ \\
\hline & & 2000 & 3 & $S$ & D & $\mathrm{D}$ & $S$ & D & $\mathrm{s}$ & $\mathbf{S}$ & $\mathbf{s}$ & D & $\mathbf{S}$ & $\mathbf{S}$ & $\mathbf{S}$ & $\mathbf{S}$ & D & $\mathrm{s}$ & $10 / 15$ \\
\hline & $\mathrm{H}$ & 80 & 4 & $\mathrm{~S}$ & D & D & D & D & D & D & $\mathbf{S}$ & D & $\mathrm{S}$ & $\mathrm{D}$ & D & D & $\mathrm{D}$ & D & $3 / 15$ \\
\hline & & 400 & 5 & D & D & S & $\mathbf{S}$ & D & D & $\mathrm{s}$ & $\mathrm{S}$ & s & D & $\mathrm{S}$ & D & D & D & D & $6 / 15$ \\
\hline & & 2000 & 6 & $\mathrm{~s}$ & D & $\mathrm{s}$ & $\mathrm{s}$ & $\mathrm{s}$ & D & D & D & $\mathbf{S}$ & $\mathbf{S}$ & $\mathrm{s}$ & $\mathbf{s}$ & $\mathbf{s}$ & $\mathbf{s}$ & $\mathbf{s}$ & $11 / 15$ \\
\hline \multirow[t]{6}{*}{22. v. 50} & $\mathrm{~K}$ & 80 & I & D & D & D & D & D & D & D & - & D & D & D & $\mathrm{s}$ & D & $\mathbf{s}$ & D & $2 / 14$ \\
\hline & & 400 & 2 & $\mathrm{~s}$ & $\mathrm{~s}$ & $\mathrm{D}$ & D & D & D & D & D & $\mathrm{s}$ & D & D & D & D & $\mathrm{D}$ & - & $3 / 14$ \\
\hline & & 2000 & 3 & D & $\mathrm{s}$ & $\mathrm{s}$ & D & - & s & - & D & s & - & D & D & D & D & D & $4 / 12$ \\
\hline & $\mathrm{H}$ & 80 & 4 & D & $\mathrm{D}$ & D & D & D & D & D & D & - & D & $\mathrm{D}$ & D & D & D & D & $0 / 14$ \\
\hline & & 400 & ธ & D & D & D & D & D & D & D & D & D & D & D & D & $\mathrm{D}$ & D & D & $0 / 15$ \\
\hline & & 2000 & 6 & D & $\mathrm{S}$ & D & D & $\mathbf{s}$ & D & D & D & S & - & $D$ & $\mathrm{D}$ & $\rightarrow$ & $\mathbf{S}$ & $\mathrm{S}$ & $5 / 13$ \\
\hline \multirow[t]{6}{*}{ 12. vi. 50} & $\mathbf{K}$ & 80 & 1 & $\mathrm{~s}$ & $\mathrm{~s}$ & $\mathrm{~s}$ & $\mathrm{~S}$ & $\mathrm{~s}$ & $\mathrm{~S}$ & $\mathrm{~s}$ & $\mathrm{~s}$ & $\mathrm{~s}$ & $\mathrm{~s}$ & $\mathrm{~s}$ & $\mathrm{~S}$ & $\mathbf{s}$ & $\mathrm{s}$ & $\mathbf{s}$ & $15 / 15$ \\
\hline & & 400 & 2 & - & $\mathrm{s}$ & $\mathrm{S}$ & $\mathrm{S}$ & $\mathrm{S}$ & $\mathrm{S}$ & $\mathrm{S}$ & $\mathrm{S}$ & $\mathrm{s}$ & $\mathrm{S}$ & $S$ & - & $\mathrm{s}$ & - & $\mathrm{S}$ & $12 / 12$ \\
\hline & & 2000 & 3 & S & S & S & S & S & D & $S$ & S & S & $S$ & $\mathrm{~S}$ & $\mathrm{~S}$ & S & $\mathrm{S}$ & $\mathbf{S}$ & $11 / 15$ \\
\hline & $\mathrm{H}$ & 80 & 4 & $\mathrm{~s}$ & s & D & $\mathrm{s}$ & s & - & S & $\mathrm{s}$ & - & $s$ & $\mathbf{S}$ & $\mathrm{s}$ & s & - & D & $10 / 12$ \\
\hline & & 400 & 5 & $\mathrm{~S}$ & $\mathrm{~S}$ & $\mathrm{~s}$ & $\mathrm{~S}$ & $\mathrm{~S}$ & s & $S$ & $\mathrm{~S}$ & $\mathrm{~S}$ & $\mathrm{~S}$ & $\mathrm{~S}$ & $\mathrm{~S}$ & $\mathrm{~S}$ & $\mathrm{~S}$ & $\mathrm{~S}$ & $15 / 15$ \\
\hline & & 2000 & 6 & $S$ & D & $s$ & $\mathrm{~S}$ & $\mathrm{D}$ & $\mathrm{s}$ & - & $S$ & $\mathbf{S}$ & $\mathrm{s}$ & $S$ & $S$ & $\mathrm{~s}$ & - & $\mathbf{S}$ & $11 / 13$ \\
\hline \multirow[t]{6}{*}{ 4. vii. 50} & $\mathbf{K}$ & 80 & 1 & $\mathrm{D}$ & D & $\mathrm{s}$ & $\mathrm{D}$ & $\mathrm{S}$ & D & D & $S$ & D & D & D & D & D & D & D & $3 / 15$ \\
\hline & & 400 & 2 & D & S & $\mathrm{D}$ & D & D & S & S & S & S & $\mathbf{S}$ & $\mathrm{S}$ & D & D & S & D & $8 / 15$ \\
\hline & & 2000 & 3 & $\mathbf{S}$ & $\mathbf{S}$ & s & $\mathrm{D}$ & $\mathbf{S}$ & $\mathbf{S}$ & D & $\mathrm{S}$ & D & $\mathbf{S}$ & s & D & $\mathbf{S}$ & $\mathrm{S}$ & $\mathbf{S}$ & $11 / 15$ \\
\hline & H & 80 & 4 & D & D & D & - & D & D & D & D & D & D & D & D & D & D & D & $0 / 14$ \\
\hline & & 400 & 5 & $\mathrm{D}$ & $\mathrm{S}$ & D & $\mathrm{S}$ & $\mathrm{s}$ & $\mathbf{s}$ & D & S & D & $\mathrm{D}$ & D & D & D & D & D & $5 / 15$ \\
\hline & & 2000 & 6 & $\mathrm{D}$ & $\mathrm{D}$ & $\mathrm{S}$ & D & D & D & $\mathbf{S}$ & D & D & $\mathrm{D}$ & $\mathbf{s}$ & D & $\mathrm{D}$ & $\mathrm{s}$ & D & $4 / 15$ \\
\hline \multirow[t]{6}{*}{ 11. vii. 50} & $\mathrm{~K}$ & 80 & 1 & D & $\mathrm{D}$ & D & D & - & D & D & D & D & $\mathrm{D}$ & D & D & $\mathrm{D}$ & D & D & $0 / 14$ \\
\hline & & 400 & 2 & D & $\mathbf{S}$ & D & D & - & D & D & D & $\mathbf{S}$ & D & $\mathbf{S}$ & D & D & D & D & $3 / 14$ \\
\hline & & 2000 & 3 & D & $\mathbf{S}$ & S & $\mathrm{s}$ & D & D & S & D & S & $\mathrm{S}$ & $\mathrm{s}$ & D & D & $\mathrm{S}$ & D & $8 / 15$ \\
\hline & $\mathrm{H}$ & 80 & 4 & $\mathrm{D}$ & $\mathrm{D}$ & $\mathrm{D}$ & $\mathrm{s}$ & D & $\mathrm{D}$ & D & S & D & D & D & D & D & D & D & $2 / 15$ \\
\hline & & 400 & 5 & D & D & D & D & D & D & D & D & D & D & D & D & D & D & D & \\
\hline & & 2000 & 6 & D & D & $\mathbf{D}$ & D & D & D & D & $\mathrm{D}$ & s & D & $\mathrm{S}$ & D & D & $\mathrm{S}$ & D & $3 / 15$ \\
\hline \multirow[t]{6}{*}{ 18. vii. 50} & $\mathbf{K}$ & 80 & 1 & D & D & D & D & S & D & D & D & D & D & D & D & D & D & D & $1 / 15$ \\
\hline & & 400 & 2 & D & $\mathrm{D}$ & $\mathrm{D}$ & D & D & D & D & $\mathrm{S}$ & S & D & D & D & D & D & D & \\
\hline & & 2000 & 3 & D & $\mathrm{S}$ & D & $\mathrm{S}$ & - & D & D & $S$ & D & D & D & $\mathbf{S}$ & $\mathbf{S}$ & $\mathrm{S}$ & D & $6 / 14$ \\
\hline & $\mathrm{H}$ & 80 & 4 & D & D & D & $\mathrm{D}$ & D & $\mathrm{D}$ & $\mathrm{D}$ & D & D & $\mathrm{D}$ & D & D & D & D & D & $0 / 15$ \\
\hline & & 400 & 5 & D & D & D & D & D & D & D & $\mathrm{D}$ & D & D & D & D & D & D & - & $0 / 14$ \\
\hline & & 2000 & 6 & D & - & D & D & D & $\mathrm{D}$ & D & - & D & D & D & D & D & D & D & $0 / 13$ \\
\hline \multirow[t]{6}{*}{ 1. viii. 50} & $\mathbf{K}$ & 80 & 1 & $\mathrm{D}$ & D & D & - & D & - & $\mathrm{D}$ & D & D & $\mathrm{D}$ & D & D & D & $\mathrm{S}$ & D & $1 / 13$ \\
\hline & & 400 & 2 & D & 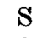 & $\mathrm{D}$ & D & - & - & S & $\mathbf{S}$ & $\mathbf{S}$ & $\mathbf{S}$ & D & $\mathbf{S}$ & - & $\mathrm{S}$ & D & \\
\hline & & 2000 & 3 & $\mathrm{~s}$ & $\mathrm{~s}$ & D & D & $\mathbf{S}$ & - & $S$ & D & $\mathrm{S}$ & $\mathbf{S}$ & $\mathrm{s}$ & - & $\mathrm{s}$ & D & D & $8 / 13$ \\
\hline & $\mathbf{H}$ & 80 & 4 & $\mathrm{D}$ & D & D & $\mathrm{D}$ & D & $\mathrm{D}$ & D & D & D & $\mathrm{D}$ & D & - & $\mathrm{D}$ & D & D & $0 / 14$ \\
\hline & & 400 & 5 & D & D & D & D & - & S & D & - & D & $\mathrm{D}$ & $\mathrm{s}$ & - & D & D & - & $2 / 11$ \\
\hline & & 2000 & 6 & D & $\mathbf{S}$ & D & $\mathbf{S}$ & D & $\mathbf{S}$ & D & D & D & $\mathbf{S}$ & D & D & $\mathbf{S}$ & $\mathrm{S}$ & - & $6 / 14$ \\
\hline
\end{tabular}

$\mathrm{D}=$ dead; $\mathbf{S}=$ survived 14 days ; $-=$ mouse died by accident, or during period of investigation. 
Table 2 shows the mean squares for litter differences and error. The overall comparison shows that the mean square for litter differences is slightly larger than the error mean square. The difference is in this direction in five out of the six tests; but does not reach the $5 \%$ level of significance.

Tables 3 and 4 give details of the results of the separate assays. There is satisfactory agreement between the results of the three methods, considering the

Table 2. Mean squares obtained for variation between litters and for 'error'

\begin{tabular}{|c|c|c|c|c|c|c|}
\hline \multirow[b]{2}{*}{$\begin{array}{l}\text { Date } \\
\text { of test }\end{array}$} & \multicolumn{2}{|c|}{ Method (i) } & \multicolumn{2}{|c|}{ Method (ii) } & \multicolumn{2}{|c|}{ Method (ini) } \\
\hline & $\begin{array}{c}\text { Between } \\
\text { litters }\end{array}$ & Error & $\begin{array}{l}\text { Between } \\
\text { litters }\end{array}$ & Error & $\begin{array}{c}\text { Between } \\
\text { litters }\end{array}$ & Error \\
\hline 11. iv. 50 & $1 \cdot 154(14)$ & $0 \cdot 874(67)$ & $1 \cdot 132(14)$ & $0.910(67)$ & $1 \cdot 141(14)$ & $0.917(67)$ \\
\hline l. v. 50 & $0.870(14)$ & $1 \cdot 119(68)$ & 0.868 (14) & $1 \cdot 102(68)$ & $0 \cdot 622(14)$ & $0.865(68)$ \\
\hline 22. v. 50 & - & - & $1 \cdot 140(14)$ & $0 \cdot 777(62)$ & $1 \cdot 193(14)$ & $0.937(62)$ \\
\hline 4. vii. 50 & $1 \cdot 253(14)$ & $0.907(69)$ & $1.307(14)$ & $0.922(69)$ & $1.328(14)$ & $0.930(69)$ \\
\hline 11. vii. 50 & - & - & $1.406(14)$ & $1 \cdot 291(68)$ & $1.508(14)$ & $1.911(69)$ \\
\hline 1. viii. 50 & $0.946(14)$ & $0.875(57)$ & 0.949 (14) & $0 \cdot 884(57)$ & $0.908(14)$ & $0.855(57)$ \\
\hline Overall & $1.056(56)$ & $0.947(261)$ & $1 \cdot 134(84)$ & $0.981(391)$ & $\mathrm{I} \cdot 117(84)$ & $1.077(391)$ \\
\hline Variance ro & & & & & & \\
\hline $5 \%$ point & & & 1 & & & \\
\hline
\end{tabular}

(Figures in brackets are the numbers of degrees of freedom.)

Table 3. Results of the separate assays, with fiducial limits $(P=0.95)$

E.D. 50 of potency ratio Method

\begin{tabular}{|c|c|c|c|c|}
\hline \multirow{2}{*}{$\begin{array}{c}\text { Date } \\
\text { test }\end{array}$} & \multirow[b]{2}{*}{ Slope } & \\
\hline & & (i) & (ii) & (iii) \\
\hline \multirow[t]{3}{*}{ 11. iv. 50} & $\mathbf{K}$ & 634 & 563 & 559 \\
\hline & H & 1,714 & 3,013 & 2,898 \\
\hline & Ratio & $2 \cdot 70$ & $5 \cdot 35$ & $5 \cdot 18$ \\
\hline \multirow[t]{3}{*}{ 1. v. 50} & $\mathrm{~K}$ & 993 & 1,069 & 1,121 \\
\hline & & 572 & 554 & 563 \\
\hline & Ratio & 0.58 & 0.52 & 0.50 \\
\hline \multirow[t]{3}{*}{ 22. v. 50} & $\mathbf{K}$ & $(18,766)$ & 2,901 & 2,196 \\
\hline & H & - & 11,940 & 7,791 \\
\hline & Ratio & - & $4 \cdot 11$ & 3.55 \\
\hline \multirow[t]{3}{*}{ 4. vii. 50} & $\mathbf{K}$ & 433 & 435 & 432 \\
\hline & $\mathrm{H}$ & $(6,244)$ & 4,190 & 3,148 \\
\hline & Ratio & $14 \cdot 4$ & $9 \cdot 62$ & $7 \cdot 30$ \\
\hline \multirow[t]{3}{*}{ 11. vii. 50} & $\mathbf{K}$ & 1,616 & 3,154 & 2,246 \\
\hline & $\mathrm{H}$ & - & 13,163 & 6,834 \\
\hline & Ratio & - & $4 \cdot 17$ & $3 \cdot 04$ \\
\hline 18. vii. 50 & $\mathbf{K}$ & 3,481 & - & 2,949 \\
\hline \multirow[t]{3}{*}{ 1. viii. 50} & $\mathbf{K}$ & 680 & 658 & 680 \\
\hline & $\mathbf{H}$ & 2,407 & 2,897 & 3,330 \\
\hline & Ratio & $3 \cdot 54$ & $4 \cdot 40$ & $5 \cdot 06$ \\
\hline
\end{tabular}

Hucial limits \% $(P=0.95)$

\begin{tabular}{|c|c|c|c|c|}
\hline \multicolumn{2}{|c|}{ (i) } & \multicolumn{2}{|l|}{ (ii) } & \multirow[t]{2}{*}{ (iii) } \\
\hline L.M. & N.L.M. & L.M. & N.L.M. & \\
\hline $25-3,400$ & $21-12,000$ & - & 一 & 44-227 \\
\hline $5-420$ & $53-500$ & - & 一 & $36-280$ \\
\hline- & - & 28-820 & $27-960$ & $27-367$ \\
\hline —* & $48-370$ & —* & - & $42-241$ \\
\hline - & $37-420$ & 一 & - & $44-227$ \\
\hline - & - & - & $25-320$ & $30-330$ \\
\hline - & $0-\infty$ & - & - & $36-275$ \\
\hline - & - & - & - & $28-353$ \\
\hline - & - & $20-2,400$ & $17-6,700$ & $22-451$ \\
\hline $34-320$ & $33-340$ & - & - & $44-226$ \\
\hline $0-\infty \dagger$ & $0-\infty \dagger$ & 一 & - & $37-274$ \\
\hline - & 一 & $24-2,200$ & $23-2,600$ & $27-364$ \\
\hline - & $51-490$ & - & - & 38-262 \\
\hline - & 一 & - & - & $31-324$ \\
\hline - & - & $11-150,000$ & $16-7,500$ & $42-413$ \\
\hline- & $32-\left(51 \cdot 1 \times 10^{5}\right)$ & — & - & $36-275$ \\
\hline-448 & $37-580$ & 一 & - & $41-440$ \\
\hline $46-1,800$ & $44-3,300$ & - & - & $34-294$ \\
\hline - & - & $30-600$ & $29-680$ & $25-399$ \\
\hline
\end{tabular}

$\dagger$ Slope not significantly different from zero.

Method (i) uses separate slopes, method (ii) a pooled slope for vaccines $K$ and $H$ in each test, method (iii) the overall pooled slope.

L.M. denotes taking account of litter-mates, N.L.M. not doing so.

The potency ratio tabulated is that of $K$ to $H$. 
magnitude of their errors. The reduction in error due to the use of litter-mates is small. A reduction of this amount, even if statistically significant would be of no practical importance. The advantage of using litter-mates might, however, be appreciable with less inbred strains of mice. There is of course a large reduction in error, when an overall mean slope is used.

The slopes in Table 4 do not differ significantly ( $\chi^{2}=7 \cdot 68$ with 9 D.F.). The $\chi^{2}$ values for departures from linearity in the dosage response relation are not abnormally high or low. One value, that from 11 July 1950 exceeds the upper $5 \%$ level

Table 4. Slopes and values of $\chi^{2}$ for departures from linearity

\begin{tabular}{|c|c|c|c|c|c|c|c|c|}
\hline \multirow{4}{*}{$\begin{array}{l}\text { Date of test } \\
\text { 11. iv. } 50\end{array}$} & \multirow{4}{*}{$\begin{array}{l}\text { Slope } \\
\mathrm{K}\end{array}$} & & \multicolumn{4}{|c|}{ Standard error of slope } & \multirow{3}{*}{\multicolumn{2}{|c|}{$\chi^{2}$ with D.F. }} \\
\hline & & \multirow[b]{2}{*}{ Slope } & \multicolumn{2}{|c|}{ Method (i) } & \multicolumn{2}{|c|}{ Method (ii) } & & \\
\hline & & & L.M. & N.L.M. & L.M. & N.L.M. & & \\
\hline & & 0.757 & $0 \cdot 324$ & $0 \cdot 346$ & - & - & $0 \cdot 148$ & 1 \\
\hline & $\mathrm{H}$ & $1 \cdot 887$ & 0.598 & $0 \cdot 640$ & - & - & $0 \cdot 133$ & 1 \\
\hline & Ratio & $1 \cdot 082$ & — & - & $0 \cdot 272$ & $0 \cdot 288$ & $2 \cdot 614$ & 3 \\
\hline I. v. 50 & $\mathrm{~K}$ & $1 \cdot 372$ & - & 0.422 & - & - & 0.011 & 1 \\
\hline & $\mathrm{H}$ & $1 \cdot 053$ & - & $0 \cdot 364$ & - & - & $0 \cdot 122$ & 1 \\
\hline & Ratio & $1 \cdot 191$ & - & - & - & $0 \cdot 274$ & $0 \cdot 462$ & 3 \\
\hline 22. v. 50 & $\mathrm{~K}$ & $0.457^{*}$ & - & $0 \cdot 400$ & - & - & 0.008 & 1 \\
\hline & $\mathrm{H}$ & - & - & - & - & - & - & - \\
\hline & Ratio & 0.929 & - & - & $0 \cdot 301$ & $0 \cdot 341$ & $4 \cdot 872$ & 3 \\
\hline 4. vii. 50 & $\mathbf{K}$ & $1 \cdot 042$ & $0 \cdot 344$ & $0 \cdot 361$ & - & - & 0.221 & 1 \\
\hline & $\mathrm{H}$ & $0.772^{*}$ & $0 \cdot 398$ & 0.418 & - & - & 4.003 & 1 \\
\hline & Ratio & 0.936 & - & - & $0 \cdot 264$ & $0 \cdot 275$ & $4 \cdot 805$ & 3 \\
\hline 11. vii. 50 & $\mathbf{K}$ & $1 \cdot 609$ & - & 0.562 & $\ldots$ & - & 0.545 & 1 \\
\hline & $\mathrm{H}$ & - & - & - & - & - & - & - \\
\hline & Ratio & 0.867 & - & - & $0 \cdot 358$ & $0 \cdot 315$ & $9 \cdot 921$ & 3 \\
\hline 18. vii. 50 & $\mathrm{~K}$ & 1.002 & - & $0 \cdot 440$ & - & - & $0 \cdot 283$ & 1 \\
\hline 1. viii. 50 & $\mathrm{~K}$ & $1 \cdot 108$ & 0.373 & 0.398 & - & - & $2 \cdot 684$ & 1 \\
\hline & $\mathrm{H}$ & $1 \cdot 458$ & 0.526 & 0.562 & - & - & $0 \cdot 541$ & 1 \\
\hline & Ratio & $1 \cdot 232$ & - & - & 0.146 & $0 \cdot 319$ & $3 \cdot 387$ & 3 \\
\hline
\end{tabular}

of significance $(P=0.02)$, but this is in no way unexpected for the greatest of seventeen values. Even the value 0.008 on 1 May 1950 is not suspiciously low $(P=0.07)$. Eleven out of the seventeen values of $\chi^{2}$ are below the average, but this might well be a chance effect ( 8.5 are expected with a standard error of $2 \cdot 1)$. If litter-mates had an appreciable effect in reducing error, the $\chi^{2}$ values should have been significantly low.

The results of this series of tests are in reasonable agreement with a large number of tests previously carried out on the same vaccines, without using litter-mates. The average fiducial limits of error $(P=0.95)$ for a test with forty-five animals

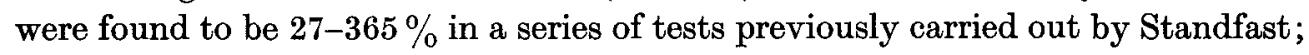


in the present series they are $34-308 \%$. The increased accuracy is due to a larger slope in the present series of tests, not to the use of litter-mates. The average overall slope in the earlier series was $0 \cdot 79 \pm 0.06$; in this series of tests it is $1 \cdot 11 \pm 0 \cdot 10$.

\section{SUMMARY}

A series of comparative assays of two pertussis vaccines was carried out by the mouse intracerebral method, using litter-mates and placing one member of each litter on each dose.

A statistical analysis of the results by the method described by Irwin (1950, p. 231), was carried out, making due allowance for the use of litter-mates. A slight increase of accuracy was found in five out of six assays, but this did not reach the $5 \%$ level of significance. Any real increase of accuracy must have been small. The results show that, as far as these tests are concerned, there is no advantage in the use of litter-mates, particularly when one takes account of the large amount of additional work necessary in the animal house.

\section{REFERENCES}

IRWIN, J. O. (1950). Biological assays with special reference to biological standards. J. Hyg., Camb., 48, 215-38.

Kendrick, P. L., Elderling, G., Dixon, M. K. \& Misner, J. (1947). Mouse protection tests in the study of pertussis vaccine. Amer. J. publ. Hlth, 37, 803 .

(MS. received for publication 28. Ix. 54) 